\title{
Mediastinal Actinomycosis. A Case-Report
}

\author{
Luciano Santana-Cabrera', Ana Casamitjana-Ortega ${ }^{1}$, José María López-Vega ${ }^{2}$, \\ Cristina Rodríguez-Escot ${ }^{1}$, Manuel Sánchez-Palacios ${ }^{1}$
}

${ }^{1}$ Department of Intensive Care, Universitary Hospital Insular in Gran Canaria, Las Palmas de Gran Canaria, Spain; ${ }^{2}$ Department of Internal Medicine, Universitary Hospital Insular in Gran Canaria, Las Palmas de Gran Canaria, Spain.

Email: lsancabx@gobiernodecanarias.org

Received September $3^{\text {rd }}$, 2011; revised October 16 ${ }^{\text {th }}$, 2011; accepted October $23^{\text {rd }}, 2011$.

\begin{abstract}
Thoracic actinomycosis is a rare disease in the era of antibiotics. Infection involving the mediastinum is extremely infrequent and the symptoms are nonspecific. It's very important to include actinomycosis in the differential diagnosis of pulmonary affections and mediastinal masses. We present a case of posterior mediastinal mass found on chest computed tomography and the patient was treated with penicillin and fully recovered.
\end{abstract}

Keywords: Mediastinal Diseases, Actinomycosis, Respiratory Insufficiency

\section{Introduction}

Actinomycosis in humans is caused predominantly by Actinomycetes israelii, a filamentous, Gram-positive anaerobic bacterium that is found in the normal flora of the oropharynx. Clinical syndromes have traditionally been divided into cervicofacial, thoracic, and abdominal infection [1]. We present a case of mediastinal actinomycosis that presented as posterior mediastinal mass on Chest computed tomography (CT).

\section{Case Report}

A 51 year old patient with a medical history of morbid obesity, hypertension, diabetes mellitus type 2, diabetic nephropathy and retinopathy, who started a month before admission with dysphagia, fever, pain in his back and progressive dyspnoea at rest, and who arrived at the Emergency Department in a state of respiratory failure requiring mechanical ventilation. Under suspicion of pulmonary thromboembolism (PTE) a CT with contrast of the pulmonary arteries (Figure 1) was performed, with no signs of PTE. It was found a soft tissue density posterior mediastinal mass, depending on oesophagus which contacted with the great vessels and the vertebral bodies, making an imprint on the back wall of the left atrium, and that did not enhance after contrast administration. It also has bilateral pleural effusion with atypical morphology, loculated and with passive segmental atelectasis. We proceeded to drain pleural effusions, resulting in a purulent fluid. It was also carry out an oesophageal endoscopy which ruled out neoplastic disease. Samples were taken by puncture-aspiration with a fine needle of the mediastinal mass and pleural fluids. The histopathology of the surgical biopsy, stained with haematoxylin and eosin, reveal multiple colonies of Actinomyces israelii, characteristic branching filaments in their periphery, surrounded by inflammatory cells and immersed into a purulent cavity. The patient was treated with penicillin and fully recovered.

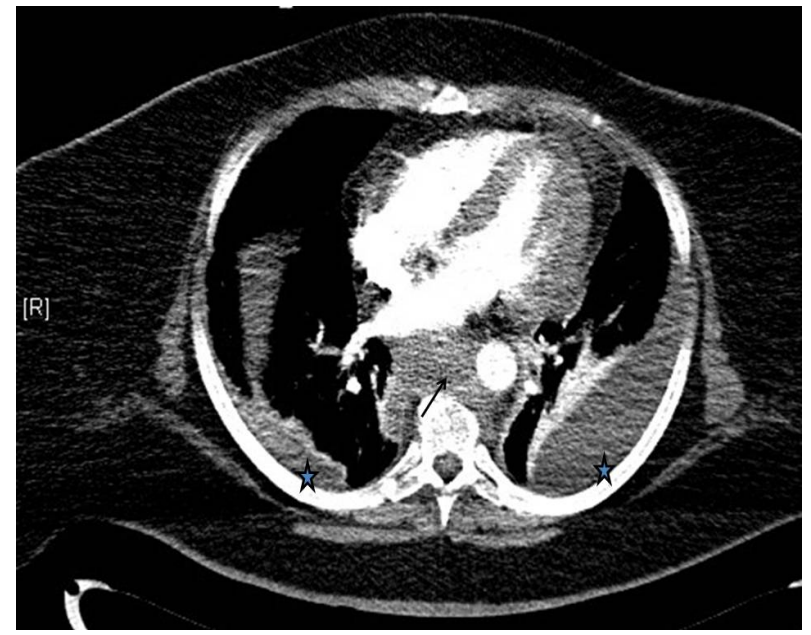

Figure 1. Chest CT with contrast in mediastinal window, which shows the posterior mediastinal mass that makes contact with the great vessels and vertebral bodies, making an imprint in the left atrium (arrow). And also bilateral pleural effusion with atypical morphology (stars). 


\section{Discussion}

Thoracic involvement is most often due to aspiration of oropharyngeal contents with bronchopulmonary invasion of the organism and subsequent contiguous spread to the chest wall or mediastinum. Infection involving the mediastinum is extremely rare and the symptoms are nonspecific and include weight loss, malaise, and coughing. Mediastinitis usually results from either perforation of the oesophagus, penetrating chest trauma, complications of thoracic surgery, tracheobronchial perforation, or contiguous spread of pulmonary infection. As the presentation of the disease is so plural, actinomycosis is often confused with neoplastic disease. The chest clinical presentation is very rare, more often primary pulmonary affectation, sometimes associated with necessitates empyema, being so much rare the mediastinal affectation [2]. Among cases of mediastinal involvement, we can distinguish those with primary pulmonary affectation with secondary mediastinal compromise of those cases of primary mediastinal involvement, as the case report that we present [3-5].

\section{Conclusions}

Infection involving the mediastinum is extremely infrequent and the symptoms are nonspecific. For this reason, it's very important to include actinomycosis in the differential diagnosis of pulmonary affections and mediastinal masses.

\section{REFERENCES}

[1] I. Krivokuca, D. W. de Lange and J. W. Lammers, “Three Patients with Thoracic Actinomycosis,” Ned Tijdschr Geneeskd, Vol. 152, No. 7, 2008, pp. 831-837.

[2] M. Enders, K. Reitnauer, M. Girmann, W. Lindemann, D. Ukena and G. W. Sybrecht, "Thoracic Actinomycosis-A Case Report,” Pneumologie, Vol. 53, No. 4, 1999, pp. 216-218.

[3] J. J. Baik, G. L. Lee, C. G. Yoo, S. K. Han, Y. S. Shim, Y. W. Kim, "Pulmonary Actinomycosis in Korea," Respirology, Vol. 4, 1999, pp. 31-35. doi:10.1046/j.1440-1843.1999.00152.x

[4] V. A. Fernández, S. R. Roca and A. L. Piñeiro, "Pulmonary Pseudotumor with Mediastinal Extensión Caused by Actinomyces,” Arch Bronconeumol, Vol. 32, No. 7, 1996, pp. 374-375.

[5] A. G. Bassiri, R. E. Girgis and J. Theodore, "Actinomyces Odontolyticus Thoracopulmonary Infections. Two Cases in Lung and Heart-Lung Transplant Recipients and a Review of the Literature,” Chest, Vol. 109, No. 4, 1996, pp. 1109-1111. doi:10.1378/chest.109.4.1109 